\title{
Comparison of Different Extraction Methods for Flavonoids and Polyphenols from Manilkara Zapota Leaves and Evaluation of Antioxidant Activity
}

\author{
Fei-Yue Ma ${ }^{a}$, Xiu-Mei Zhang ${ }^{b,}{ }^{*}$, Yu-Ge Liu ${ }^{c}$, Qiong Fu ${ }^{d}$, Zhi-Ling Ma ${ }^{f}$ \\ Key Laboratory of Tropical Fruit Biology, Ministry of Agriculture, South Subtropical Crop \\ Research Institute, Chinese Academy of Tropical Agricultural Science (CATAS), Zhanjiang \\ 524091, Guangdong, China \\ a mfyflc@yahoo.com, ${ }^{\mathrm{b}}$ asiazhang1975@163.com, ${ }^{\mathrm{c}}$ liuyugehb@126.com, ${ }^{\mathrm{d}}$ bingqi1986@163.com, ${ }^{\mathrm{f}}$ \\ mbelle@163.com
}

Keywords: Manilkara zapota leaves, Total phenols, Total flavonoids, Antioxidant activity.

Abstract. In the present research, a comparative study was conducted between heat reflux extraction (HRE), ultrasonic-assisted enxtraction (UAE) and negative pressure cavitation extraction (NPCE) for extract total phenols and total flavonoids from Manilkara zapota leaves. Single-factor experiments were applied to optimize the operating parameters of different extraction method. The optimum parameters were: HRE, extract temperature $70^{\circ} \mathrm{C}, 50 \%$ ethanol, liquid/solid ratio $30: 1$, extract time 2h. Furthermore, the antioxidant activities of extracts from HRE, UAE and NPCE were 0.110, 0.094, $0.164 \mathrm{mg} / \mathrm{mL}\left(\mathrm{IC}_{50}\right)$, according to the $\mathrm{DPPH}$ radical-scavenging assay.

\section{Introduction}

Manilkara zapota is a nutritious, fleshy berry, with a scurfy brown peel and light brown, brownish yellow to reddish brown pulp, with a texture varying from gritty to smooth. The pulp has a very sweet pleasant flavor. The fruit and its peel contain high amounts of saponin, which has astringent properties similar to tannin ${ }^{[1]}$. Manilkara zapota had the highest oxygen radical absorbance capacity and total phenols content among many fruits ${ }^{[2]}$. In the aqueous extract of Manilkara zapota, it has a significant higher reducing power and DPPH activity ${ }^{[3]}$. But, there is scant report on extracting active compounds from Manilkara zapota leaves.

In the present work, the important parameters involved in different extraction process were optimized by single-factor experiments. The effect of different extraction method on the extraction yields of flavonoids and phenols from Manilkara zapota leaves were comparative. The present research would be helpful for the further explore and full use of the renewable resource leaves of Manilkara zapota.

\section{Materials and methods}

\subsection{Materials and reagents}

The leaves of Manilkara zapota collected from South Subtropical Crop Research Institute. The material was dried in the shade, powdered by a disintegrator (HX-200A, Yongkang Hardware and Medical Instrument Plant, China). Ethanol obtained from Tianjin Chemical Reagents Co. (Tianjin, China) was analytical grade. Rutin and Gallic acid were purchased from Sigma-Aldrich (Steinheim, Germany).

\subsection{Different methods extraction procedure and optimized parameters}

In order to investigate the effects of flavonoids and phenols of Manilkara zapota leaves on different extraction methods, a comparative study was conducted between HRE, UAE and NPCE after the optimization of the latter. The experiments of determination total flavonoids and total phenols content were carried out according to the method of Jin et al ${ }^{[4]}$ and Asma Hamood et al ${ }^{[5]}$. In addition, the antioxidant activity of extract was evaluated. 


\subsection{1, 1-Diphenyl-2-picrylhydrazyl (DPPH) radical scavenging activity assay}

The antioxidant activities of samples were studied using the 1, 1-diphenyl-2-picrylhydrazyl (DPPH) radical scavenging assay. The experiment was carried out according to the method of Ma et al ${ }^{[6]}$.

\section{Result and discussion}

\subsection{Optimization of HRE parameters}

Heat reflux extractions (HRE) were performed in a water-bath that allows variation of temperature. After optimization, the optimal conditions of HRE were as follows: extraction time $2 \mathrm{~h}$, liquid/solid ratio 30:1 (w/w). Extract temperature and ethanol concentration were important parameters for the extraction yields of flavonoids and phenols from Manilkara zapota leaves. The effects of ethanol concentration on the flavonoids and phenols extraction yields were studied at the range of $30 \%-80 \%$. As can be seen from Fig. 1A, the extraction yield of total flavonoids and total phenols increased drastically with increasing ethanol concentration from 30\% to 50\%. Then it decreased obviously with increasing ethanol concentration. It indicated that $50 \%$ ethanol was enough for flavonoids and phenols to be extracted.

Generally, the extraction temperature was an important factor contributing to increased extraction efficiency. As shown in Fig. 1B, the extraction yield increased with increasing temperature. Hence, temperature $70^{\circ} \mathrm{C}$ was chosen for the optimized parameters.
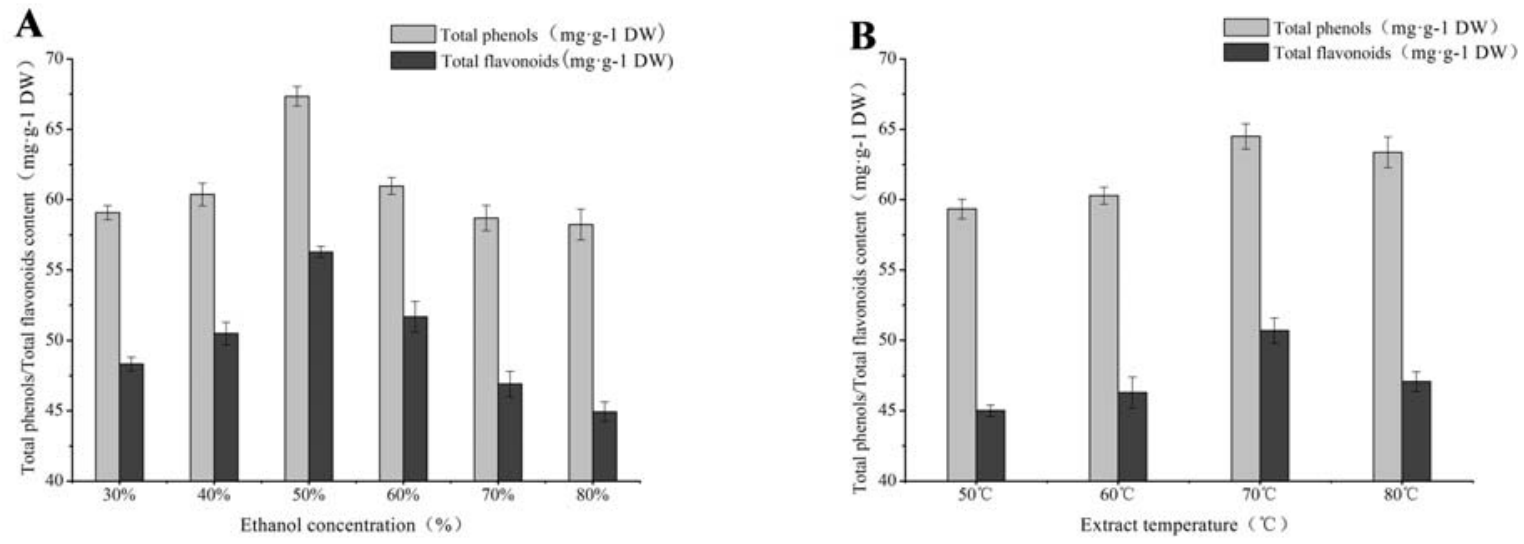

Fig. 1. Effects of the ethanol concentration and extract temperature on the extraction yields of flavonoids and phenols from Manilkara zapota leaves with HRE(A and B).

\subsection{Optimization of UAE parameters}

In order to select the optimized conditions, ethanol concentration and extraction time were investigated. In Fig. 2A, it was indicated that extraction time had slender effects on the extraction yields of flavonoids and phenols from Manilkara zapota leaves. The effects of ethanol concentration on UAE the extraction yields were shown Fig. 2B. The highest extraction yields of flavonoids and phenols from Manilkara zapota leaves could be observed at $50 \%$ ethanol. The optimized conditions of UAE were: liquid/solid ratio 30:1 (w/w), 50\% ethanol, extraction time 40min. 

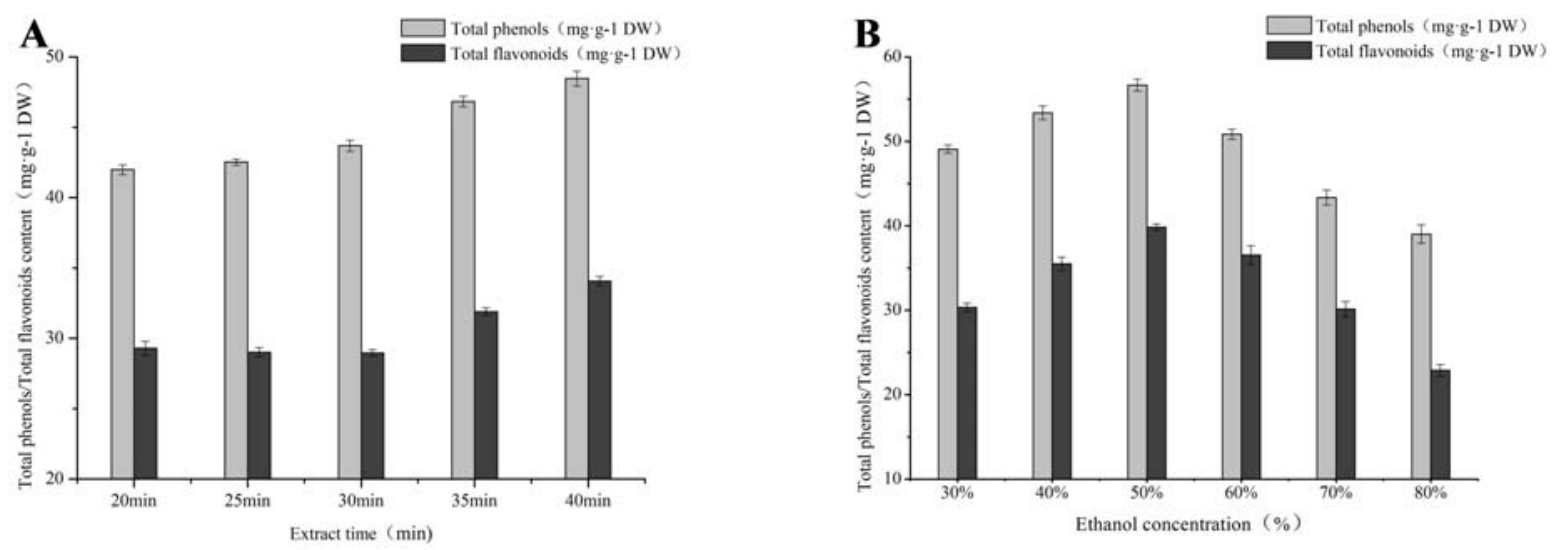

Fig. 2. Effects of the extract time and ethanol concentration on the extraction yields of flavonoids and phenols from Manilkara zapota leaves with UAE (A and B).

\subsection{Optimization of NPCE parameters}

Negative pressure cavitation extraction (NPCE), a novel extraction technology, was applied for active secondary metabolites extraction ${ }^{[7]}$. The method extraction procedure was carried out according to the method of Wei, et al ${ }^{[7]}$. To obtain appropriate extraction conditions flavonoids and phenols from Manilkara zapota leaves, a series of studies were conducted to investigate the effect of NPCE conditions on their extraction yields. From Fig. 3A, B and C, the optimal conditions of NPCE were as follows: liquid/solid ratio 30:1 (w/w), 50\% ethanol, extraction time $30 \mathrm{~min}$, negative pressure $0.060 \mathrm{MPa}$. Therefore, for obtaining active secondary metabolites, it can be concluded that NPCE was more suitable for industrial scale-up applications than the aforementioned methods.
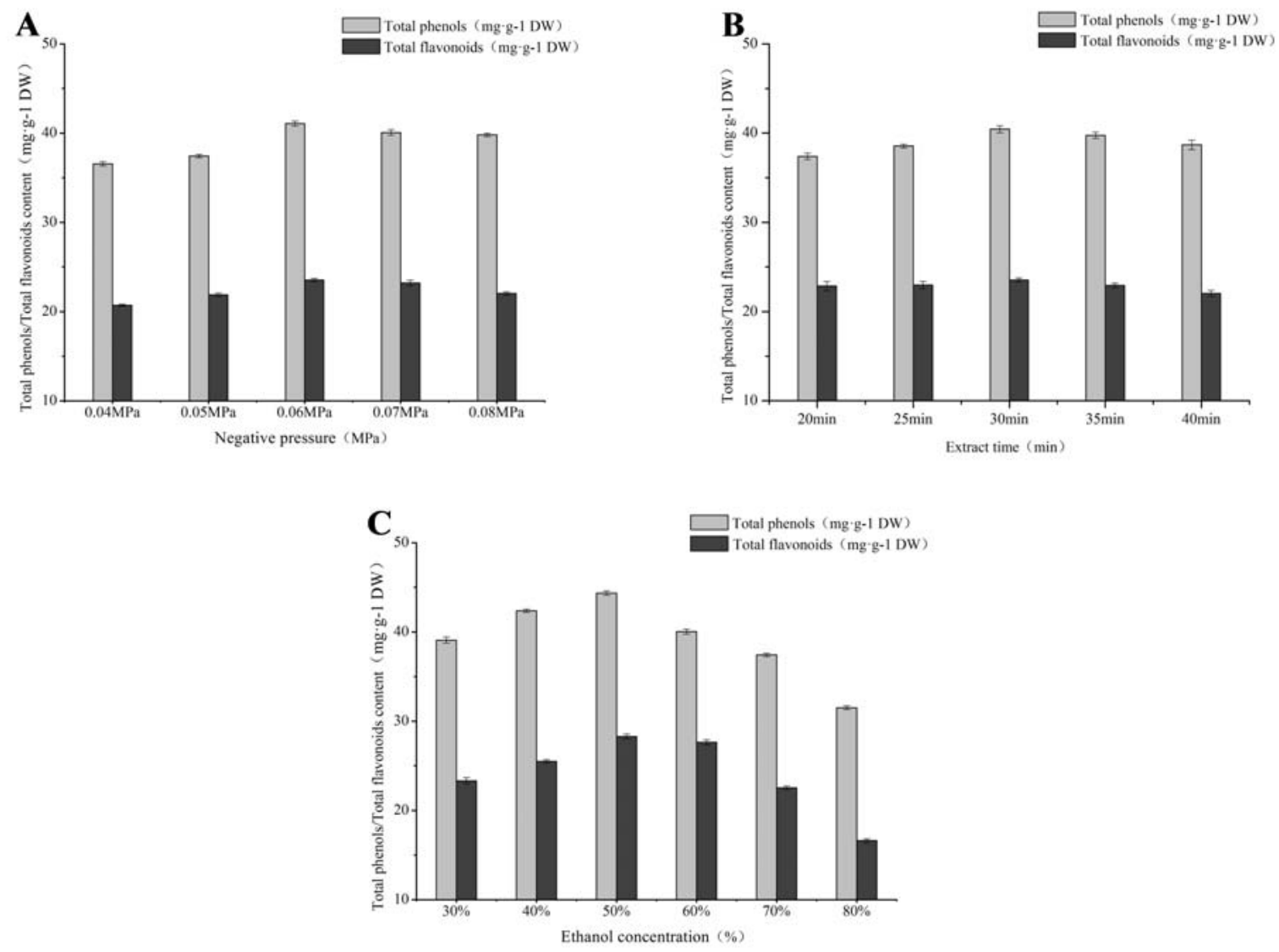

Fig. 3. Effects of the negative pressure, extract time and ethanol concentration on the extraction yields of flavonoids and phenols from Manilkara zapota leaves with NPCE(A, B and C). 


\subsection{DPPH radical scavenging activity assay}

The results of DPPH scavenging activity are shown in Fig. 4. The concentration of the sample required to cause a $50 \%$ decrease in initial $\mathrm{DPPH}$ free radical concentration $\left(\mathrm{IC}_{50}\right)$ was used as an index to compare the antioxidant activity. It was observed UAE extract from Manilkara zapota leaves exhibited potent DPPH radical-scavenging activity with an $\mathrm{IC}_{50}$ value of $0.094 \mathrm{mg} / \mathrm{mL}$, which was higher than HRE extract $(0.110 \mathrm{mg} / \mathrm{mL})$ and NPCE extract $(0.164 \mathrm{mg} / \mathrm{mL})$. From these results, the flavonoids and phenols extraction yields were high, but there were not the strongest DPPH radical-scavenging activity. Therefore, for obtaining natural antioxidants, it can be concluded that UAE was more suitable than the other methods.

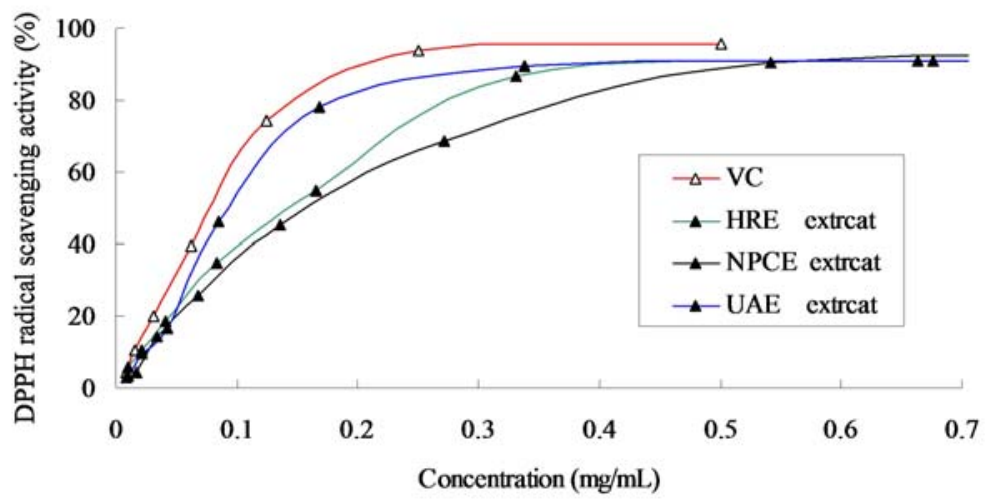

Fig. 4. DPPH radical scavenging activity of HRE extracts, UAE extracts and NPCE extracts.

\section{Conclusion}

In the present study, compared to other extraction method, the extraction yield of flavonoids and phenols from Manilkara zapota leaves for HRE was highest. The optimum parameters were: HRE, extract temperature $70^{\circ} \mathrm{C}$, 50\% ethanol, extract time $2 \mathrm{~h}$, liquid/solid ratio 30:1. For DPPH radical-scavenging activity, UAE was more suitable than the other methods. $\mathrm{IC}_{50}$ value for the extract of HRE, UAE and NPCE were $0.110 \mathrm{mg} / \mathrm{mL}, 0.094 \mathrm{mg} / \mathrm{mL}$ and $0.164 \mathrm{mg} / \mathrm{mL}$, respectively. Moreover, it was proved that the optimised NPCE process was scalable by pilot-scale application.

\section{Acknowledgements}

This work was financially supported by the Fund on Basic Scientific Research Project of Nonprofit Central Research Institutions (1630062015011).

\section{References}

[1] S. K. Devatkal, R. Kamboj, D. Paul: J. Food Sci. Technol. (2014) 51(2):387-391.

[2] M. Isabelle, L.L. Bee, T.L. Meng, K. Woon-Puay, H. Dejian, N.O. Choon: Food Chem. (2010) 123:77-84.

[3] K.S. Jamuna, C.K. Ramesh, T.R. Srinivasa, K.L. Raghu: J. Pharm. Res. (2010) 3:2378-2380.

[4] S. Jin, M. Yang, Y. Kong, X.H. Yao, Z.F. Wei, Y.G. Zu, Y.J. Fu: Chin. Tradit. Herb Drugs (2011) 42(11):2235-2239.

[5] A.H. Al-Saeedi, M.A. Hossain: Asian Pac. J. Trop. D. (2015) 5(4):316-321.

[6] F.Y. Ma, C. B. Gu, C.Y. Li, M. Luo, W. Wang, Y.G. Zu, J. Li, Y.J. Fu: Sep. Purif. Technol. (2013) 115: 136-144. 
[7] Y. Kong, Z.F. We, Y.J. Fu, C.B. Gu, C.J. Zhao, X.H. Yao, T. Efferth: Food Chem. (2011) 128: 596-605. 